\title{
Identification of anti-tumoral feedback loop between VHLa and hnRNPA2B1 in renal cancer
}

\author{
Yanbin Liu', Hui Zhang ${ }^{1}$, Xingzhi Li' ${ }^{2}$ Changming Zhang ${ }^{3}$ and Haide Huang ${ }^{3}$
}

\begin{abstract}
Our previous study identified a novel VHLa isoform which negatively modulated hnRNPA2B1 expression and therefore influenced pyruvate kinase transcript splicing in renal cancer, while the regulation and initiation of alternative translation are largely unknown. Here we unraveled the CUG-mediated translation start of VHLa, which was subjected to the regulation by both eukaryotic initiator factor eIF2A and RNA helicase elF4A. Unexpectedly, we found hnRNPA2B1 promoted VHLa alternative translation as well via direct interaction with its octadic pentamer region of VHL transcript. The N-terminal of VHLa was indispensable in mediating ubiquitination of hnRNPA2B1 at lysine residues 274 and 305. We further identified aberrant overexpression of c-myc as upstream oncogenic signaling to positively regulate hnRNPA2B1 transcription in renal cancer. Therefore, our data suggested an anti-tumoral feedback loop between VHLa and hnRNPA2B1.
\end{abstract}

\section{Introduction}

VHL is well-recognized as essential tumor suppressor gene, and dysfunction of which intimately associates with malignancy-predisposing von Hipple-Lindau disease ${ }^{1,2}$. Assembling investigations uncover that loss-of-function of VHL via loss of alleles ${ }^{3}$, somatic mutation or epigenetic inhibition ${ }^{4}$ accounts for the majority of clear cell renal cell carcinomas (ccRCCs) incidence clinically ${ }^{5}$. Functionally, VHL constitutively associates with elongins $B$ and $C$ to form a relative stable ternary complex ${ }^{6}$, which further serves as adapter mediating the assembly of VCB-CUL2$\mathrm{RBX}^{7,8}$ with characteristic ubiquitin E3 ligase activity against $\alpha$-subunit of hypoxia inducible factors (HIF1 $\alpha$ and HIF $2 \alpha)^{9}$. HIF $\alpha$ accumulation in the context of VHLdeficiency provokes array of oncogenic signaling, most of which link to acute or chronic hypoxic responses ${ }^{10-12}$. Therapeutics targeting VHL-HIF-VEGF pathway emerge

\footnotetext{
Correspondence: Yanbin Liu (yanbliu@hotmail.com)

${ }^{1}$ Institute of Immunology and Molecular Medicine, Jining Medical University, Jining, China

${ }^{2}$ Department of Urological Surgery, Longgang District People's Hospital of

Shenzhen, Shenzhen, China

Full list of author information is available at the end of the article

Edited by S. Inoue
}

as standard procedure in treatment of metastatic renal cell carcinoma $^{13}$.

The coding region of VHL across three exons and encodes two proteins: VHL-24 and VHL-19. The latter initiates from an internal in-frame ATG codon and represents the predominant and conservative isoform ${ }^{14-16}$. Our pervious study uncovered the characteristic perinuclear distribution of VHL-19 complexing with the ribosomal large subunit 6 (RPL6), which may play critical roles in quality control of nascent peptides ${ }^{17}$. In addition, we identified a novel translation variant of VHL as well, termed VHL $\alpha$, via alternative translation mechanism, which negatively regulates heterogenous nuclear ribonucleoprotein A2B1 (hnRNPA2B1) and therefore modulates pyruvate kinase transcription splicing ${ }^{18}$. However, the initiation site and regulation of VHL $\alpha$ alternative translation are still largely unknown. In view of well-recognized CUG-mediated alternative translation ${ }^{19-21}$, here we further pinpointed the initiation site of VHL $\alpha$ through serial synonymous mutations. The potential involvements of eukaryotic translation initiation factor 2A (eIF2A) which was previously indicated playing important roles in CUGinitiated translation ${ }^{20}$ are addressed in this study. We also attempt to systematic investigate the possible 
contributions of RNA helicases in VHL $\alpha$ translation with shRNA-mediated gene silencing.

Our previous study demonstrated that VHL $\alpha$ decreased hnRNPA2B1 at protein level which was efficiently blockaded by proteasome inhibitor, MG132, indicating the potential ubiquitination of hnRNPA2B1 by VHL $\alpha^{18}$. In this study, we set out to further identify the ubiquitination sites of hnRNPA2B1 and critical regions of VHL $\alpha$ responsible for protein interaction. Noting worthily, we also found that ectopic over-expression of hnRNPA2B1 stimulated short-term increase of VHL $\alpha$ translation (unpublished data), which suggested a potential post-transcriptional regulation of $\mathrm{VHL} \alpha$ by hnRNPA2B1 in turn. As a ubiquitous RNA binding protein $^{22}$, the essential biological roles of hnRNPA2B1 are increasingly unraveled. Here we unraveled the mutual regulation between VHL $\alpha$ and hnRNPA2B1, which constitutes feedback loop in malignancy of ccRCC.

\section{Materials and methods}

\section{Cell culture and transfection}

Human kidney cell HEK293T and ccRCC cell lines 786-0, 769-p, ACHN, A498, A704, Caki-1, Caki-2 and RCC4 were obtained from the American Typical Culture Collection (ATCC, NY, USA). Cell identities were authenticated by short tandem repeat (STR) profiling and regularly examined mycoplasma contamination by PCR method. HEK293T cells were cultured in DMEM high glucose and other cells in RPMI modified medium, all mediums were supplemented with $10 \%$ fetal bovine serum (FBS) and $1 \%$ penicillin/streptomycin. Cell culture was maintained in humidified $\mathrm{CO}_{2}$ incubator (5\%). Transfection was performed with Lipofectamine 2000 (Invitrogen, MO, USA) in accordance with manufacturer. All shRNAs were purchased from Origene (Beijing, China) and overexpressing plasmids for VHL, hnRNPA2B1, c-myc, HAeIF2A, HA-eIF4A were ordered from Vigenebio (Jinan, China).

sheIF2A-1: 5'-CAGCCTTACACTACTTCTAAAGAT GGCAC-3';

sheIF2A-2: 5 '-TACAAGGTGGCTGTCTATGTTCCA GGAAG-3';

shNT: 5'-GCACTACCAGAGCTAACTCAGATAG

TACT-3';

shDed1: 5'-ACTCATCCGAAATGGACGTGACT

TCTTAT-3';

shDHX29: 5'-ATTGAAGATGCCATGACCAATACAC

TCTT-3';

shRHA: 5'-ACCGAGGAGCCAACTTGAAGGATTAC TAC-3';

shVASA: 5'-GAGGACTCCATCTTTGCACATTATCA GAC-3';

sheIF4A-1: $5^{\prime}$-AGCAGCGAGCCATTCTACCTTGTA TCAAG-3';
sheIF4A-2: $\quad$ '-ATTGGCTCAGCAGATACAGAAGG TGGTCA-3';

shc-myc: 5'-GAGGATATCTGGAAGAAATTCGAGC TGCT-3';

shhnRNPA2B1: 5'-GCTACGGAGGTGGTTATGACA ACTATGGA-3'.

\section{Clinical samples}

Totally, 30 ccRCC tumor samples were collected from Longgang District People's Hospital from 20015 to 2019 during radical surgery, and pathologically examined by three independent pathologists. The fresh tissues were flash frozen in liquid N2 until use. The written informed consents were acquired from all enrolled subjects and this study was approved by Institutional Ethics Committees of Longgang District Hospital.

\section{Western blots}

Cell lysates were prepared in RIPA lysis buffer on ice and debris were completely removed by refrigerated centrifugation. One microgram of plasmids were used for transfection and protein and was harvested $24 \mathrm{~h}$ later unless specified. For drug treatments in 786-0 cells, $100 \mu \mathrm{M}$ Aurin tricarboxylic acid was applied for 0, 6, 12, $24 \mathrm{~h}$, different dosage of Acriflavine $(0,10,20,30 \mu \mathrm{M})$ was applied for $4 \mathrm{~h}$, Pateamine A $(15 \mu \mathrm{M})$, Hippuristanol $(5 \mu \mathrm{M})$ and Silvestrol $(20 \mathrm{nM})$ were applied individually for 0,12 , and $24 \mathrm{~h}$, respectively. In induced expression of hnRNPA2B1, Doxycycline $(0,0.1,0.2,1,2 \mu \mathrm{M})$ was applied for $4 \mathrm{~h}$ or $0.5 \mu \mathrm{M}$ of Doxycycline was applied for 0 , $6,12,18$, and $24 \mathrm{~h}$, respectively. All proteins were resolved with 12\% SDS-PAGE and transferred onto PVDF membrane (Millipore, MO, USA) on ice. 5\% skim milk in TBST buffer was used for brief blocking and followed by incubation with primary antibody (VHL $\alpha$ antisera were prepared in mice as previously described ${ }^{18}$; rabbit anti$\beta$-actin, 1:2000, \#4967; rabbit anti-VHL, 1:1000, \# 68547; rabbit anti-eIF2A, 1:1000, \#5324; rabbit anti-eIF4A, 1:1000, \#2425 from Cell Signaling Technology, MA, USA; mouse anti-FLAG, 1:2500, F3165; mouse anti-HA, 1: 5000, H9658; rabbit anti-hnRNPA2B1, 1: 2500, HPA001666; rabbit anti-Myc tag, 1: 500, 06-549; rabbit anti-c-myc, 1: 1000, C3956 from Sigma, MO, USA) overnight at $4{ }^{\circ} \mathrm{C}$. After wash for $30 \mathrm{~min}$, the membrane was hybridized with HRP-conjugated secondary antibodies (goat anti-rabbit, 1: 5000, \#7074; horse anti-mouse, 1: 5000, \#7076; Cell Signaling Technology, MA, USA) for another hour. The blots were visualized using the enhanced chemiluminescence kit (ECL, Millipore, MO, USA).

\section{Real-time PCR}

Total RNA extraction from either indicated tissues samples or cells was performed with TRIzol method 
(Invitrogen, MA, USA) following the provider's protocol. RNA quality was examined with Bioanalyzer 2100 (Agilent, CA, USA) prior to use. Reverse transcription with $1 \mu \mathrm{g}$ RNA was performed with High Capacity cDNA Reverse Transcription Kit (Applied Biosystems, CA, USA). The SYBR Green Real-Time Master Mixes (ThermoFisher, MA, USA) was used for quantitative PCR on CFX96 Touch Real-Time PCR Detection System (BioRad, CA, USA). The primer sequences used were listed as follows:

eIF2A Forward primer: 5'-CCGCTCTTGACAGTC CGAG-3';

eIF2A Reverse primer: 5'-GCAGTAGTCCCTTGTTAG

TGACA-3';

eIF4A Forward primer: 5'-AAGGCGTCATCGAGAGT

AACT-3';

eIF4A Reverse primer: 5'-ATGTGGCCGTTTTCCCA GTC-3';

VHL-24 Forward primer: 5'-GCAGGCGTCGAAGAG TACG-3';

VHL-24 Reverse primer: 5'-CGGACTGCGATTGCAG AAGA-3';

VHL-19 Forward primer: 5'-GGAGCCTAGTCAA GCCTGAGA-3';

VHL-19 Reverse primer: 5'-CATCCGTTGATGTGCA ATGCG-3';

VHL $\alpha$ Forward primer: 5'-CGCGTTCCATCCTCTAC CGA-3';

VHL $\alpha$ Reverse primer: 5'-TCTTCGACGCCT GCCTCCT-3';

c-myc Forward primer: 5'-GGCTCCTGGCAAAAGG

TCA-3';

c-myc Reverse primer: 5'-CTGCGTAGTTGTGCTG ATGT-3';

hnRNPA2B1 Forward primer: 5'-TGGAGGTAGC CCCGGTTATG-3';

hnRNPA2B1 Reverse primer: 5'-GGACCGTAGTTAG AAGGTTGCT-3';

$\beta$-actin Forward primer: $5^{\prime}$-CATGTACGTTGCTATCC AGGC-3';

$\beta$-actin Reverse primer: $5^{\prime}$-CTCCTTAATGTCACGC ACGAT-3'.

\section{Immunohistochemistry (IHC)}

The ccRCC tissues were embedded in paraffin blocks and sectioned into $5 \mu \mathrm{m}$ slices following the standard protocol. The slides were then deparaffinized in xylene for $5 \mathrm{~min}$ by two times and rehydrated in absolute alcohol for 3 min by two times, $95 \%, 70 \%, 50 \%$ alcohol for 3 min each. The IHC staining procedure was performed with BiotinStreptavidin HRP Detection Systems (ZSGB-BIO, Beijing, China) following the manufacturer's protocol. Endogenous peroxidase activity was blockaded with $3 \% \mathrm{H}_{2} \mathrm{O}_{2}$ solution in methanol at room temperature for $10 \mathrm{~min}$.
Antigen retrieval was achieved by heating in citrate buffer (10 mM, pH 6.0) using microwave. After blocking with $10 \%$ FBS, tissue slides were applied with indicated primary antibody (VHL $\alpha$ antisera, 1:20. Rabbit anti-myc, 1:25, HPA055893; rabbit anti-hnRNPA2B1, 1: 50, HPA001666; rabbit anti-eIF2A, 1:200, HPA036256 from Sigma, MO, USA. Rabbit Anti-eIF4A, 1:100, ab31217 from Abcam, Cambridge, UK) in humidified chamber at room temperature for $1 \mathrm{~h}$. The biotin-labeled secondary antibody was then incubated for $30 \mathrm{~min}$ and HRP-streptavidin conjugates were applied for another $15 \mathrm{~min}$ at room temperature. Chromogenic reaction was performed with diaminobenzidine (DAB) reagent. Representative images were acquired under DMi8 Inverted Microscope (Leica, Wetzlar, Germany).

\section{RNA pulldown}

RNA pulldown assay was performed with Pierce Magnetic RNA-Protein Pull-Down Kit (ThermoFisher, MA, USA) in accordance with manufacturer's instruction. All biotin-labeled RNA fragments were synthesized by Synobio-Tech (Suzhou, China). VHL-19, VHL-24, and VHL $\alpha$ transcripts were prepared with MEGAscript T7 Transcription Kit (ThermoFisher, MA, USA) following the manufacturer's manual and biotin-labeled with Pierce RNA 3' End Biotinylation Kit (ThermoFisher, MA, USA). Briefly, biotin-labeled RNA (100 pmol) was captured by $50 \mu \mathrm{L}$ of Streptavidin Magnetic Beads for $30 \mathrm{~min}$ at room temperature, and incubated with $100 \mu \mathrm{g}$ of cell lysate in RIPA lysis buffer for $60 \mathrm{~min}$ at $4{ }^{\circ} \mathrm{C}$ with agitation. The pull-downed protein species were eluted with Elution buffer for $0 \mathrm{~min}$ at $37^{\circ} \mathrm{C}$ with agitation, and subjected to western blots analysis.

\section{RNA immunoprecipitation (RIP)}

RNA-IP assay was performed with Imprint RNA Immunoprecipitation Kit (Sigma, MO, USA) according to the manufacturer's manual. Briefly, $5 \mu \mathrm{g}$ of hnRNPA2B1 antibody was immobilized on $20 \mu \mathrm{L}$ of Protein A Magnetic Beads for $30 \mathrm{~min}$ at room temperature, then incubated with cell lysates $\left(10^{6}\right.$ cells $/ 20 \mu \mathrm{L}$ lysis buffer $)$ overnight at $4{ }^{\circ} \mathrm{C}$ with rotation. RNA was recovered with TRIzol $(500 \mu \mathrm{L}$ per RIP reaction) methods and resuspended in $20 \mu \mathrm{L}$ of RNase-free water. Enrichments of target transcripts were analyzed by real-time PCR as previously described.

\section{Co-immunoprecipitation (Co-IP)}

Indicated cells were collected in ice-cold PBS with scraper and lysed in $500 \mu \mathrm{L}$ of IP lysis buffer $(50 \mathrm{mM}$ HEPES, $\mathrm{pH} 7.5,150 \mathrm{mM} \mathrm{NaCl}, 1 \mathrm{mM}$ EDTA, $2.5 \mathrm{mM}$ EGTA, 0.1\% (w/v) Tween20, $1 \mathrm{mM}$ dithiothreitol, $1 \mathrm{mM}$ $\mathrm{NaF}$ and $100 \mu \mathrm{M}$ PMSF) on ice for $30 \mathrm{~min}$. Protein concentration was determined with BCA Protein Assay Kit 
(ThermoFisher, MA, USA), and $2 \mathrm{mg}$ of protein (in $500 \mu \mathrm{L}$ of lysis buffer) was incubated with $2 \mu \mathrm{g}$ of rabbit IgG for $1 \mathrm{~h}$ at $4{ }^{\circ} \mathrm{C}$ for clearance purpose, followed by incubation with anti-FLAG M2 Magnetic Beads overnight at $4{ }^{\circ} \mathrm{C}$ with agitation. Immunoprecipitated complex was recovered with SDS-PAGE sample buffer and analyzed by western blots.

\section{Immunofluorescence}

786-0 cells were plated on cover slips and cultured overnight for attachment. Cells were fixed with $4 \%$ PFA for 15 mins and permeabilized with $0.2 \%$ Triton X-100 for $10 \mathrm{~min}$. Anti-hnRNPA2B1 (HPA065537, 1:400, Sigma, MO, USA) and VHL $\alpha$ antisera (1:20) were then incubated at $4{ }^{\circ} \mathrm{C}$ overnight after brief blocking with $5 \%$ BSA, and followed by hybridization with fluorescent secondary antibody (anti-rabbit 488, 1:200; anti-mouse 594, 1:200; ThermoFisher, MA, USA). The coverslips were mounted with ProLong Gold Antifade Mountant with DAPI (ThermoFisher, MA, USA) on glass slides. The images were acquired with confocal microscope (Leica, Wetzlar, Germany).

\section{Chromatin immunoprecipitation (ChIP)}

The indicated cells $\left(10^{6}\right)$ were first fixed with formaldehyde (1\%) at room temperature for $10 \mathrm{~min}$ and lysed in $0.5 \mathrm{~mL}$ of ice-cold lysis buffer supplemented with Protease Inhibitor Cocktail (Roche, Basel, Switzerland) for $15 \mathrm{~min}$. Cell nuclear were collected in $0.5 \mathrm{~mL}$ of nuclear lysis buffer and subjected to sonication to shear crosslinked DNA to 200-1000 bp fragments. After centrifugation, the supernatant was incubated with $2 \mu \mathrm{g}$ of cmyc antibody (AHO0062, ThermoFisher, MA, USA) and $20 \mu \mathrm{L}$ of well-dispersed protein $\mathrm{G}$ magnetic beads overnight at $4{ }^{\circ} \mathrm{C}$ with rotation. Immunoprecipitated complex was collected, and enriched DNA fragments were recovered by protease $\mathrm{K}$ digestion at $62^{\circ} \mathrm{C}$ for $2 \mathrm{~h}$ with shaking and purified using spin column. The presence of hnRNPA2B1 promoter fragments were analyzed by realtime PCR. The primer sequences used for ChIP analysis were indicated in figure legend.

\section{Luciferase reporter assay}

The exponential HEK293T cells were seeded into 6-well plate $\left(5 \times 10^{5} /\right.$ well $)$. The hnRNPA2B1 promoter $(\sim 1 \mathrm{~kb})$ luciferase reporter plasmid was ordered from GeneCopoeia (Guangzhou, China). Point-directed mutagenesis was employed to generate putative c-myc-recognizing site mutants. The luciferase plasmids $(1 \mu \mathrm{g})$ were cotransfected with either empty control or c-mycexpressing plasmids $(1 \mu \mathrm{g})$ into HEK293T cell (6-well plate) for $24 \mathrm{~h}$, and relative luciferase activities were measured with Bright-Glo Luciferase Assay System (Promega, WI, USA) on BioTek Synergy 2 Multi-Mode
Microplate Reader (BioTek, VT, USA). The mutagenesis PCR primer sequences were listed as below:

Forward: $\quad 5^{\prime}$-TGCGCGACCGTTTCTTCGCACCC TTGCGCCAAGGCAG-3';

Reverse: 5'-CTGCCTTGGCGCAAGGGTGCGAAGA AACGGTCGCGCA-3'.

\section{Cell counting kit-8 (CCK-8) assay}

The indicated cells at log phase were inoculated in 96well plate (1000 cells/well) and cultured up to 4 days. Cell viability was determined with CCK-8 kit (Dojindo, Dalian, China) following the manufacturer's instruction at day 0 , $1,2,3$, and 4 , respectively. Ten microliter of CCK-8 solution was added into each well and incubated for $2 \mathrm{~h}$ in $\mathrm{CO} 2$ incubator. Absorption was measured with microplate reader (BioTek, VT, USA).

\section{Colony formation}

The indicated cells $\left(10^{4} /\right.$ well) were plated in 6-well plate and subjected to continuous culture in regular medium for up to 10 days. Fresh culture medium was replaced every 3 days. After fixation with 4\% PFA (15 min at RT) and staining with crystal violet $(10 \mathrm{~min}$ at $\mathrm{RT})$, the resultant colonies were counted under microscopic and images were captured with DMi8 Inverted Microscope (Leica, Wetzlar, Germany).

\section{Transwell assay}

The polycarbonate membrane cell culture inserts (Coring, NY, USA) were employed to measure cell invasive capacity. The single-cell suspension prepared from indicated cells via trypsin digestion (cells $/ \mu \mathrm{L}$ ) and $100 \mu \mathrm{L}$ of cells (in serum-free medium) were seeded into the upper inserts with $750 \mu \mathrm{L}$ of complete medium supplied in lower compartments. After cultured for $12 \mathrm{~h}$, the invaded cells were fixed with 4\% PFA for $15 \mathrm{~min}$ and stained with crystal violet $(0.25 \%)$ for $30 \mathrm{~min}$. The images were captured, and cells were counted under microscope.

\section{Wound healing assay}

Wound healing assay was employed to evaluate cell migrative capacity. The indicated cells $\left(2 \times 10^{5}\right)$ were inoculated in 6-well plate and allowed for attachment overnight. The scratch was created with sterile tips and detached cells were aspirated with PBS wash. Cells were cultured up to $48 \mathrm{~h}$ and gap closure was continuously monitored under light microscope.

\section{Xenograft tumor}

The indicated cells $\left(10^{6}\right.$ cells $)$ were prepared as singlecell suspension into $100 \mu \mathrm{L}$ of PBS: Matrigel Basement Membrane Matrix (1: 1, Corning, NY, USA), and inoculated into the lower flanks of BALB/c Nude (4-weeks-old, Vitalriver, Beijing, China). The xenograft tumor growth 
was continuously monitored up to 18 days postinoculation and mice were sacrificed by cervical dislocation. Tumor volume was estimated as (Length $\times \mathrm{Width}^{2}$ )/ 2. Mice were equally divided into six groups: empty vector, c-myc-overexpressing, c-myc-overexpressing plus hnRNPA2B1-silencing, c-myc-overexpressing plus VHL $\alpha$-overexpressing, hnRNPA2B1-overexpressing, hnRNPA2B1-overexpressing plus VHL $\alpha$-overexpressing, 5 mice for each group. The animal study was approved by the Institutional Animal Care and Use Committee of Jining Medical University. Experimental mice were housed in specific pathogen-free (SPF) environment and all procedures were in strict compliance with $\mathrm{NIH}$ guideline.

\section{Statistical analysis}

Data presented in this study were analyzed and processed using Prism 8 (GraphPad, CA, USA). Unpaired student $t$-test was employed for statistical comparison between groups. The $p$ value was calculated and $p<0.05$ was considered as statistically significant.

\section{Results}

\section{eIF2A and elF4A involve in VHLa alternative translation}

Our previous study first uncovered the alternative translation of VHL transcript and identified a novel protein isoform named $\mathrm{VHL} \alpha^{18}$. However, the alternative translation initiation codon was still to be defined and the molecular mechanism underlying regulation of alternative translation of VHL transcript was largely elusive. In view of the widely recognized leucine-initiator tRNA-mediated translation start at CUG codon, we hypothesized that similar mechanism might operate in control of VHL $\alpha$ translation after close inspection of the $5^{\prime}$ region of $\mathrm{VHL}$ transcript. All 5 synonymous mutations introduced to $\mathrm{CTG}^{43}$ codon completely abolished VHL $\alpha$ expression (Fig. 1a). On the contrary, synonymous mutations affecting the neighboring codons including $\mathrm{AGG}^{43}$, $\mathrm{ATC}^{37}, \mathrm{CTT}^{40}, \mathrm{CGC}^{46}, \mathrm{ACG}^{49}$, and $\mathrm{CGC}^{52}$ manifested none of noticeable impacts on VHL $\alpha$ translation (Fig. 1a, lower). Via point-directed mutations, we provided evidence the alternative translation of VHL $\alpha$ initiated from $\mathrm{CTG}^{43}$ codon. We then further clarified the potential involvement of eukaryotic translation initiation factor $2 \mathrm{~A}$, eIF2A, in this scenario, which was previously suggested playing crucial roles in mediating CUG-initiated translation. To this end, we established ectopic eIF2Aoverexpressing (Fig. 1b, left) and eIF2A-silencing (Fig. 1b, right) cell lines and assessed the influences on VHL $\alpha$ translation. As shown in Fig. 1c, endogenous VHL $\alpha$ was increased in eIF2A-proficient cells in comparison with empty vector control, while significantly inhibited by shRNA-mediated knockdown of eIF2A. This result suggested the indispensable role of eIF2A contributing to
VHL alternative translation. Furthermore, we employed aurin tricarboxylic acid (ATA) to efficiently suppress translation start at the AUG codon and enhance CUGmediated initiation. ATA treatment significantly promoted VHL $\alpha$ expression in a time-dependent manner (Fig. 1d), while showed no evident effects on VHL transcript levels (Fig. 1e). On the other hand, acriflavine greatly compromised VHL $\alpha$ expression in a dosedependent manner (Fig. 1f), which was consistent with previous report about inhibitory action of acriflavine on CUG initiation ${ }^{20}$. Similarly, the transcript abundance of VHL was not altered by acriflavine treatment (Fig. 1g), which implicated a regulation at post-transcriptional level.

As we described previously, the octadic pentamers of VHL transcript highly likely led to formation of complex secondary structure, which hindered translation scanning and necessitated RNA helicase activities for efficient protein synthesis. To clarify the potential involvements of RNA helicases in regulation of VHL translation, we effectively silenced eIF4A, Ded1, DHX29, RHA, and VASA individually (Fig. 1h). VHL $\alpha$ was slightly upregulated only in the context of eIF4A knockdown (Fig. 1i). To further solidify our observation, we generated eIF4Aoverexpressing cells and specifically silenced eIF4A with two independent shRNAs (Fig. 1j). VHL $\alpha$ protein was significantly induced by eIF4A-depletion and reduced by ectopic eIF4A (Fig. 1k). We further employed eIF4Aspecific inhibitors including pateamine $\mathrm{A}^{23}$, hippuristanol $^{24}$ and silvestrol ${ }^{25}$, all of which showed stimulatory effects on VHL $\alpha$ translation in a time-dependent manner (Fig. 11). To exclude possible influences of eIF4A inhibitors on VHL transcription, we also analyzed mRNA and confirmed the consistent level of VHL transcripts (Fig. $1 \mathrm{~m})$. Therefore, our data suggested that eIF4A played important roles in promoting VHL translation and therefore suppressed VHL $\alpha$ expression, which critically depended on its RNA helicase activities. In addition, we analyzed relative expression of eIF2A and eIF4A regarding VHL $\alpha$ status in clinical tumors by both IHC analysis and real-time PCR, and noticed significant upregulated eIF2A and suppressed expression of eIF4A in VHL $\alpha$-positive samples (Fig. 1n). Taken together, our results identified the CUG-initiated VHL $\alpha$ translation, which was subjected to modulation by both eIF2A and eIF4A.

\section{Direct association between hnRNPA2B1 and octadic pentamer region of VHLa transcript}

Our previous study demonstrated that hnRNPA2B1 was significantly decreased by VHL $\alpha$ other than other isoforms, which was blockaded by proteasome inhibitor, MG132, and suggested the involvement of ubiquitination/ proteasome system-mediated protein degradation ${ }^{18}$. We also noticed that MG132 treatment caused increase of VHL $\alpha$ in addition to accumulation of hnRNPA2B1, which 


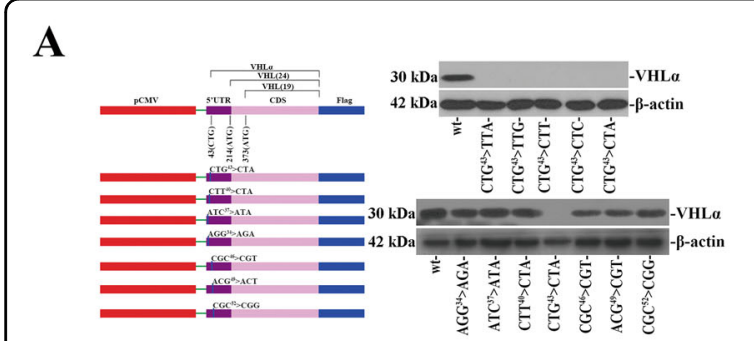

C

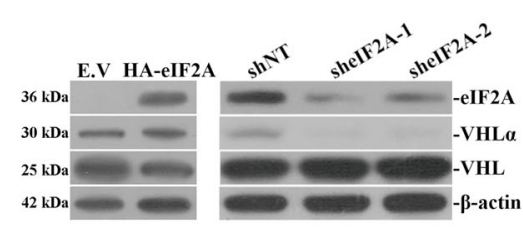

B
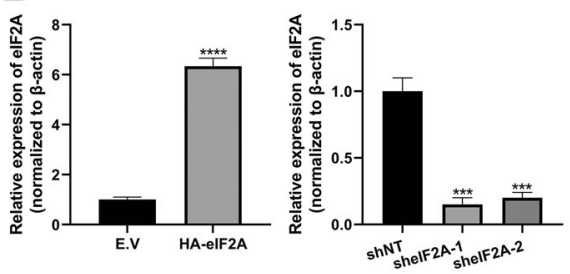

$\mathbf{E}$

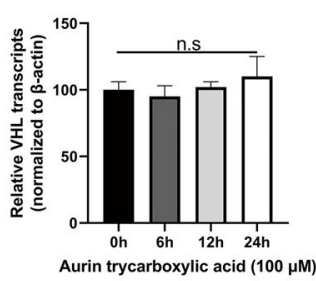

F

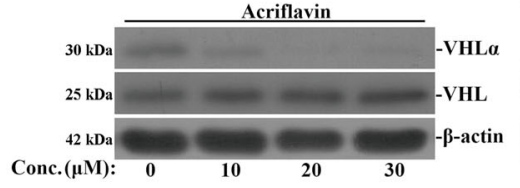

$\mathbf{J}$

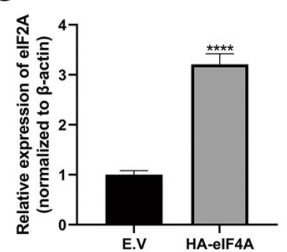

D

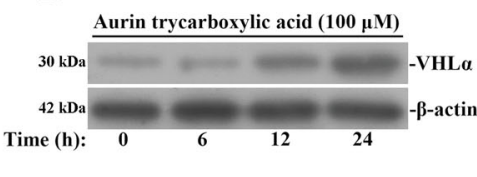

H

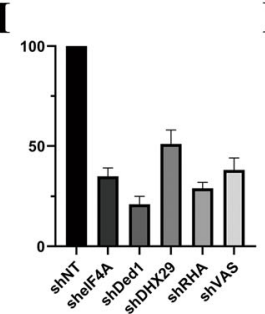

I

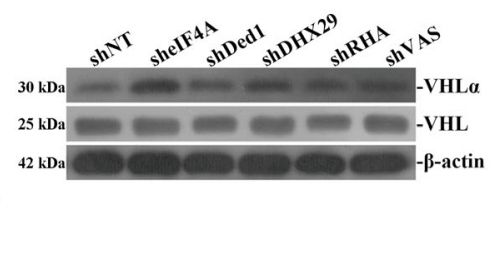

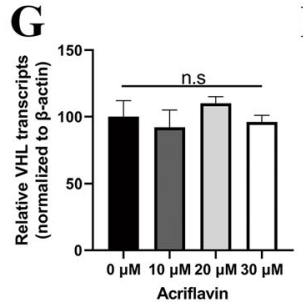

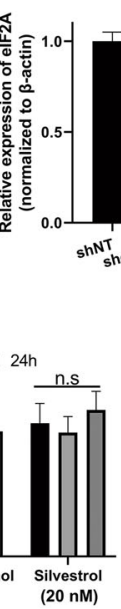

\section{K}

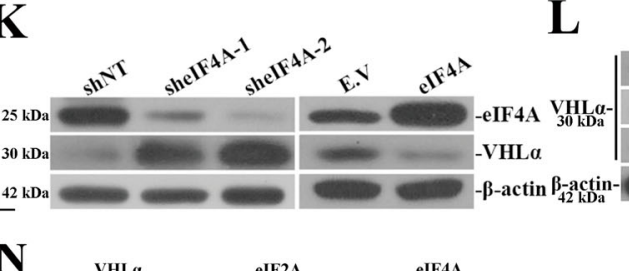

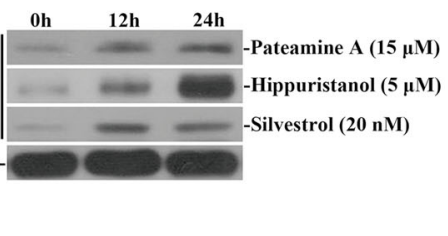

\section{M}
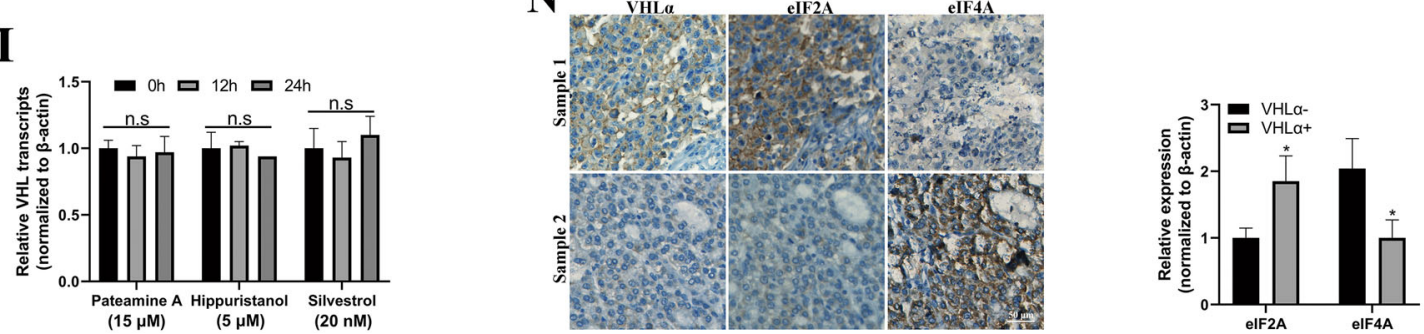

Fig. 1 elF2A and elF4A involve in VHLa alternative translation. a Immunoblots analysis of ectopic VHLa in 293T cells in response to synonymous mutations introduced at $\mathrm{CTG}^{43}$ (left) and neighboring codons $\left(\mathrm{AGG}^{34}>\mathrm{AGA}, \mathrm{ATC}^{37}>\mathrm{ATA}, \mathrm{CT}^{40}>C \mathrm{CTA}, \mathrm{CGC}^{46}>C G \mathrm{CG}, \mathrm{ACG}{ }^{49}>\mathrm{ACT}\right.$ and $\mathrm{CGC}^{52}>C G \mathrm{CG}$, right). b Quantitative PCR measurements of relative expression of elF2A in established stable cells (786-0 with empty vector (E.V) or HA-elF2A, 769-p with shNT, shelF2A-1 and shelF2A-2). c Immunoblots analysis of VHLa in response to elF2A overexpression (left) or knockdowns (right) in 293T. d Time course of VHLa induction in 293T cells by aurin tricarboxylic acid (ATA, $100 \mathrm{aM}$ ) treatments for 0, 6, 12, 24 h. e VHL mRNA transcript levels were determined by real-time PCR in ATA-treated $293 \mathrm{~T}$ cells at $0,6,12$, and $24 \mathrm{~h}$. $\mathbf{f}$ Immunoblots analysis of VHLa and VHL in response to different concentrations of scriflavin treatments $(0,10,20,30 \mathrm{M})$ for $4 \mathrm{~h}$ in $293 \mathrm{~T}$ cells. $\mathbf{g}$ VHL mRNA transcript levels were determined by real-time PCR in acriflavin-treated 293T cells as indicated in $\mathbf{f}$. $\mathbf{h}$ Knockdown efficiencies of DNA helicases including elF4A, Ded1, DHX29, RHA, and VASA were evaluated by real-time PCR. i. VHLa protein was examined in DNA helicase-deficient 293 T cell by western blots. $\mathbf{j}$ Establishment of elF4Aoverexpressing (HA-elF4A) and elF4A-silencing (shelF4A-1 and shelF4A-2) cell lines was validated by real-time PCR. $\mathbf{k}$ VHLa protein alterations upon elF4A overexpression and knockdown were evaluated by immunoblotting in 293T cells. I Relative VHLa proteins were examined in 293T in response to elF4A-specific inhibitors for 0,12 , and $24 \mathrm{~h}$ (pateamine $\mathrm{A}, 15 \mu \mathrm{M}$; hippuristanol $5 \mu \mathrm{M}$; silvestrol $20 \mathrm{nM}$ ). $\mathbf{m}$ Consistent VHL transcript abundance in response to elF4A inhibitors treatments as described in I. was measured by real-time PCR. $\mathbf{n}$ Representative IHC images of VHLa- and VHLa+ renal tumors (left) and analysis of relative expression of elF2A and elF4A regarding VHLa status by real-time PCR. Data are presented as mean \pm S.D for three independent experiments. n.s., no significance; ${ }^{*} P<0.05$; ${ }^{* *} P<0.01$; ${ }^{* * *} P<0.001$; ${ }^{* * *} P<0.0001$. 


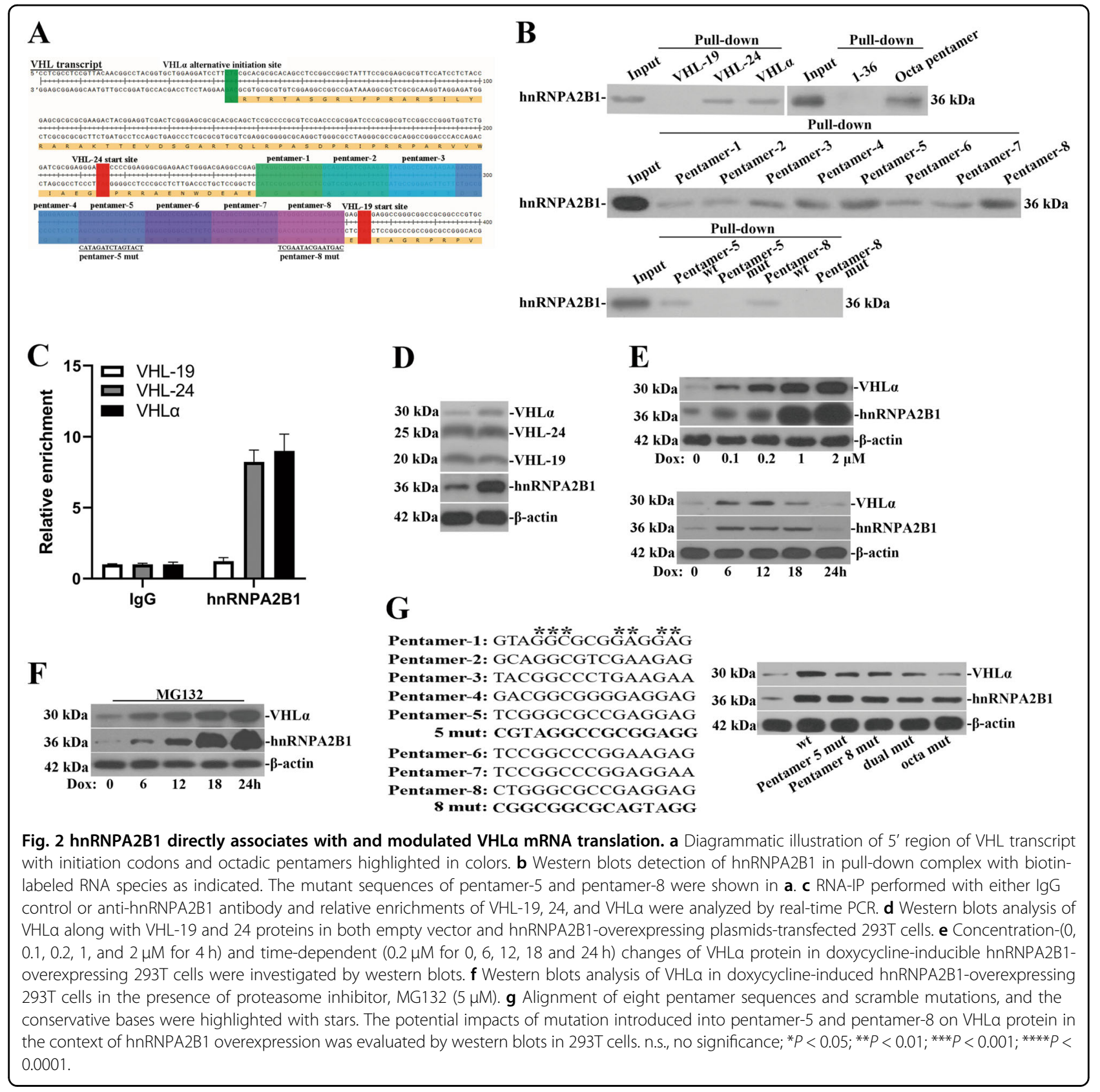

implicated the possible feedback regulation on VHL $\alpha$. In view of the ubiquitous affinity of hnRNPA2B1 to RNA species, here we set out to clarify the physical association between hnRNPA2B1 protein and VHL transcript. The characteristic features of $5^{\prime}$ VHL transcript were illustrated in Fig. 2a, with start sites of VHL-19, VHL-24, VHL $\alpha$ and octadic pentamers were highlighted in colors, and pentamer- 5 and pentamer- 8 mutant sequences were provided as well. The biotin-labeled pulldown assay demonstrated that hnRNPA2B1 complexed with both VHL-24 and VHL $\alpha$ transcript except for VHL-19, suggesting the recognizing site might locate between VHL-19 and VHL-24 start sites (Fig. 2b, upper left). The fragmentation RNA pull-down assay showed that octadic pentamer was responsible for interacting with hnRNPA2B1 (Fig. 2b, upper right). Therefore, we examined the eight pentamer sequences individually and found all of them showed affinity to hnRNPA2B1, while among of which pentamer- 5 and pentamer- 8 were the most significant ones (Fig. 2b, middle). Scramble mutations completely abolished the presence of hnRNPA2B1 in pentamer- 5 and pentamer- 8 pull-downed protein species (Fig. 2b, lower). We also performed RNA immunoprecipitation with hnRNPA2B1 antibody and observed 


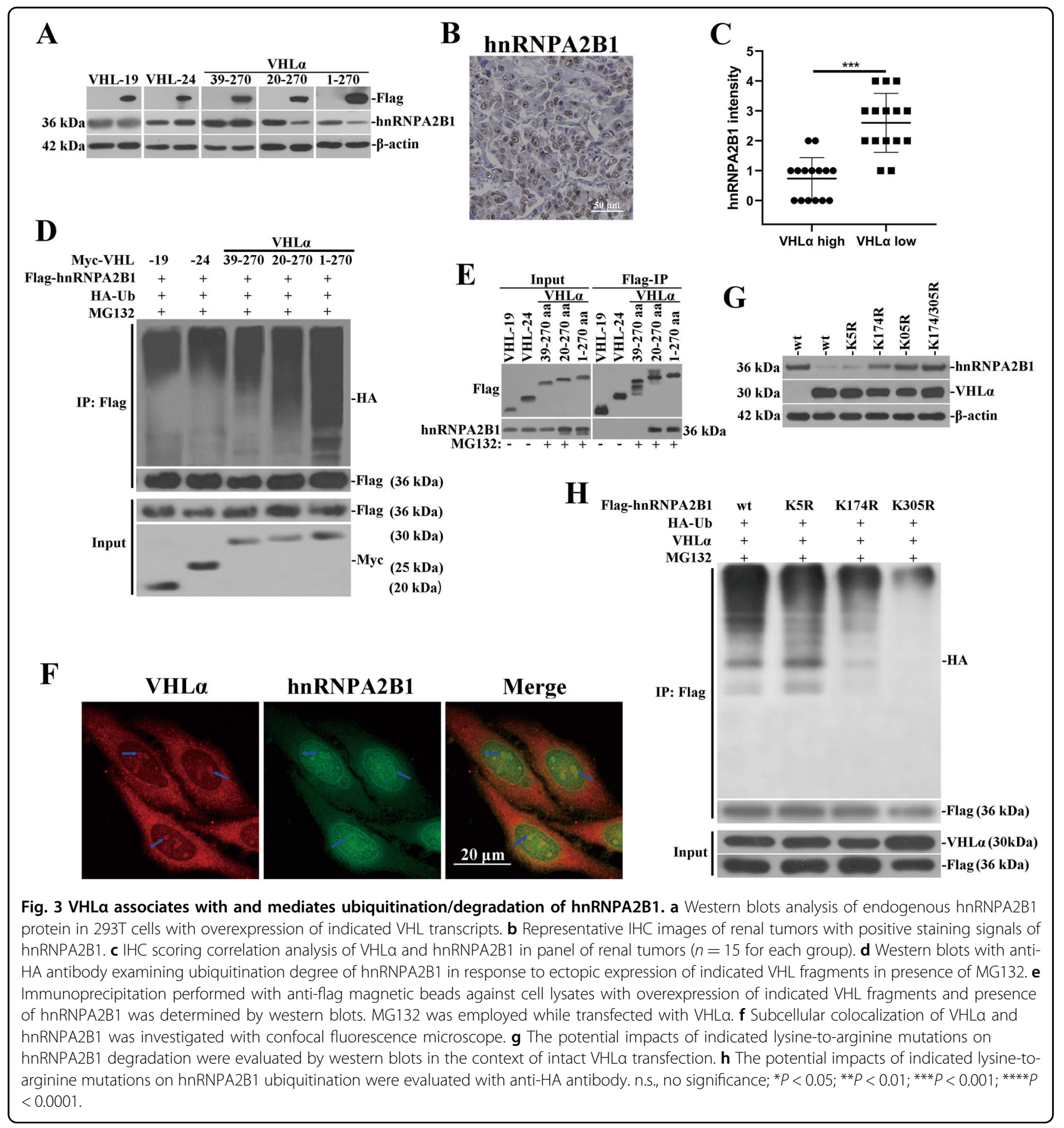

significant enrichments of VHL transcripts which were detected by VHL-24 and VHLo-specific primers (Fig. 2c). These data provided solid evidences supporting the direct interaction between hnRNPA2B1 and VHL transcripts.

To further address the possible regulation of VHL $\alpha$ translation by hnRNPA2B1 in return, we ectopically overexpressed hnRNPA2B1 for a short-term and noticed increase of VHL $\alpha$ protein (Fig. 2d). In doxycyclineinduced overexpressing system, VHL $\alpha$ increased with
Dox in a dose-dependent manner (Fig. 2e). However, long-term exposure to Dox led to decreases of both VHL $\alpha$ and hnRNPA2B1 despite of increases at early stage (Fig. $2 \mathrm{e}$, lower), which hinted that accumulated VHL $\alpha$ promoted hnRNPA2B1 ubiquitination and degradation, and which eventually compromised the stimulatory effects on VHL $\alpha$ translation. Addition of MG132, which stabilized hnRNPA2B1 and therefore continuously stimulated VHL $\alpha$ translation up to $24 \mathrm{~h}$ in our system (Fig. 2f). This 
stimulation was greatly dependent on recognition of pentamer sequences especially pentamer- 5 and pentamer8 by hnRNPA2B1, which was consolidated by the observation that scramble mutations disrupting either pentamer-5 or pentamer-8 compromised VHL $\alpha$ translation. Dual mutations of both pentamer- 5 and pentamer- 8 greatly suppressed VHL $\alpha$ expression, while disruption of all eight pentamer sequences with scramble mutation almost completely abrogated the regulation of VHL $\alpha$ by hnRNPA2B1 (Fig. 2g). Taken together, our data suggested that hnRNPA2B1 positively regulated VHL $\alpha$ translation via direct association with its octadic pentamer region.

\section{VHLa associates with and mediates ubiquitination and degradation of hnRNPA2B1}

Our previous study demonstrated VHL $\alpha$ other than both VHL-19 and VHL-24 was capable of degrading hnRNPA2B1 in renal cancer cells. The serial truncate constructs manifested that 1-38 amino acid of VHL $\alpha$ was critical for degradation of hnRNPA2B1, as endogenous

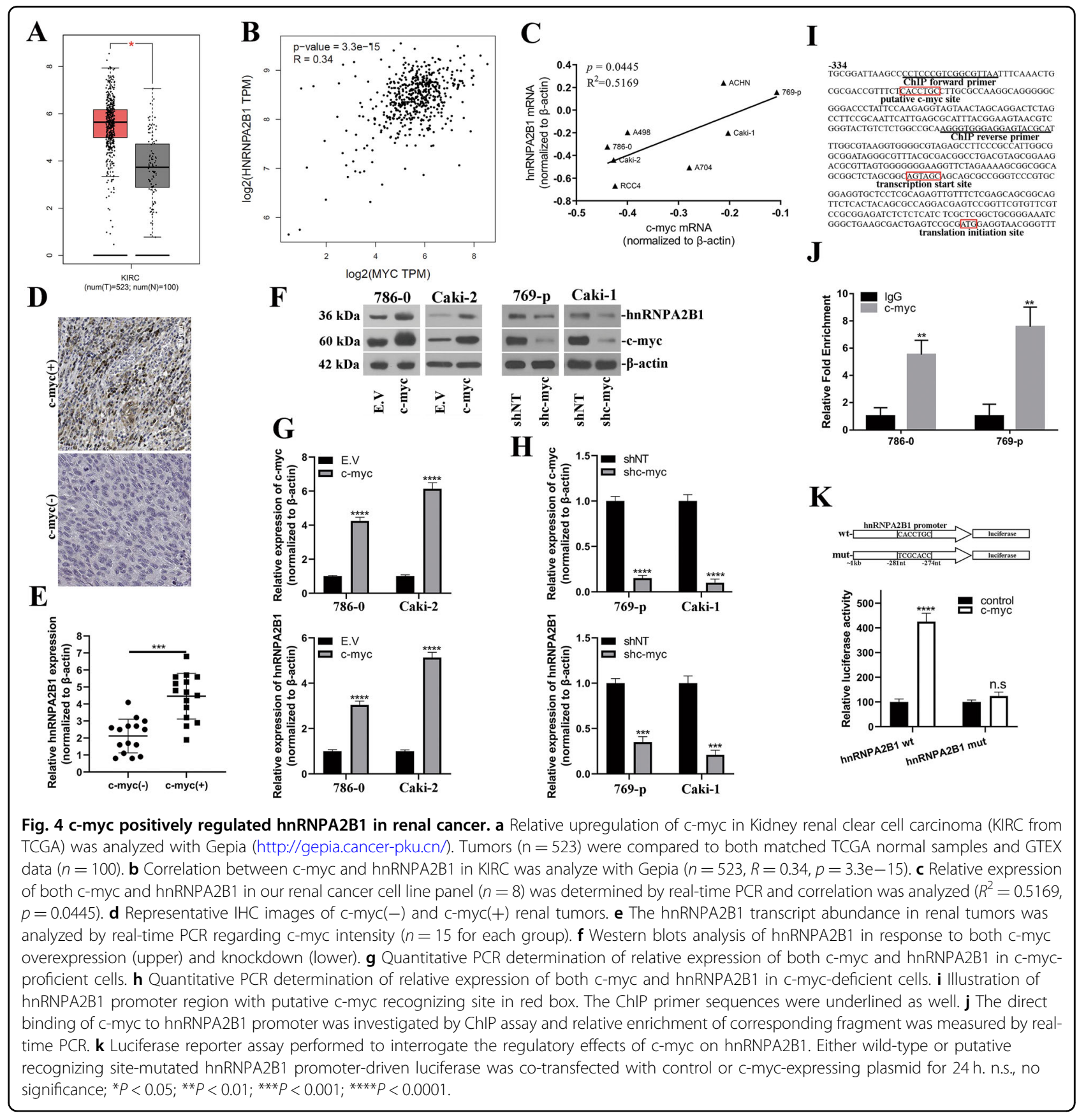


hnRNPA2B1 was significantly decreased by ectopic introduction of 20-270aa or 1-270aa VHL $\alpha$ but maintained unchanged in response to fragment 39-270 (Fig. 3a). Here we further showed the downregulation of hnRNPA2B1 in VHL $\alpha$ high-expressed renal tumor tissues, with representative IHC staining results of hnRNPA2B1 along with statistical results were provided in Fig. 3b, c. The immunoprecipitated hnRNPA2B1 was ubiquitinated to greater extent in the presence of MG132 while 293T cells were co-transfected with either fulllength of VHL $\alpha$ or 20-270aa fragment in comparison with 39-270 fragment, VHL-19 or VHL-24, respectively (Fig. 3d). Consistently, we detected hnRNPA2B1 in immunoprecipitation complex against either full-length VHL $\alpha$ or 20-270 fragment (Fig. 3e). Our data suggested that the amino acid 1-38 of VHL $\alpha$ was critically for association with and ubiquitination of hnRNPA2B1. The direct interaction between hnRNPA2B1 and VHL $\alpha$ protein was further consolidated by the observation of partial subcellular co-localization in cell nuclear, which was highlighted by blue arrows in confocal images (Fig. 3f).

Next, we attempted to identify the ubiquitination site of hnRNPA2B1 by VHL $\alpha$. Sequence analysis performed with UbPred algorithm (http://www.ubpred.org/) predicted that lysine residue-5, residue-174, and residue-305 as potential ubiquitination sites. We then employed mutagenesis PCR method to generate site-directed mutants of hnRNPA2B1 plasmids. Co-transfection with VHL $\alpha$ led to tremendous decrease of hnRNPA2B1 (wild-type and K5R), which was greatly canceled by K174R and K305R mutation individually or in combination (Fig. 3g). Consistently, the mutations at K174 and $\mathrm{K} 305$ other than K5 compromised the ubiquitination level of hnRNPA2B1 (Fig. 3h). Therefore, our data identified lysine residue 174 and 305 as ubiquitination sites by VHL $\alpha$ in renal cancer cells.

\section{c-myc positively regulated hnRNPA2B1 in renal cancer}

Our previous data uncovered the self-limiting regulation of hnRNPA2B1 via feedback loop with VHL $\alpha$ in renal cancer cell. Here we sought to identify upstream regulatory mechanism at transcriptional level. Via datamining TCGA, we found significantly higher expression of cmyc in renal tumors and positive correlation with hnRNPA2B1 (Fig. 4a, b). This stringent correlation was validated by real-time PCR in our renal cancer cell line panel including 786-0, 769-p, Caki-1, Caki-2, ACHN, A498, A704, and RCC4 (Fig. 4c, $R^{2}=0.5169, p=0.0445$ ). We further analyzed relative abundance of hnRNPA2B1 in renal tumor tissues regarding c-myc status, and tremendous upregulation of hnRNPA2B1 transcripts were noticed in c-myc $(+)$ tissues in comparison with c-myc $(-)$ group (Fig. 4d, e). These data implicated a potentially regulatory effect of c-myc on hnRNPA2B1 transcription.
Ectopic overexpression of c-myc greatly induced upregulation of hnRNPA2B1 at both protein and transcript levels, while shRNA-mediated knockdown of c-myc downregulated hnRNPA2B1 (Fig. 4f-h). With aid of bioinformatics tool (promo_v3), we predicted the putative c-myc site in promoter region of hnRNPA2B1 which was illustrated in Fig. 4i along with primer sequences used in ChIP assay. The significant enrichments of hnRNPA2B1 transcripts in immunoprecipitation complex with anti-cmyc antibody were observed in both 786-0 and 769-p cell (Fig. 4j). The regulatory effect of c-myc on hnRNPA2B1 transcription was further interrogated by luciferase reporter assay, wherein co-expression of c-myc greatly stimulated hnRNPA2B1 promoter-driven luciferase activities, which was readily abolished by scrambled mutation introduced into the putative c-myc-recognizing site (Fig. 4k). Therefore, our results clearly demonstrated hnRNPA2B1 transcription was subjected to c-myc in renal cancer cells.

\section{VHLa exerted anti-tumoral roles through negative control of oncogenic hnRNPA2B1 proteins in renal cancer}

Our previous results suggested that endogenous hnRNPA2B1 expression was modulated both by c-myc at transcriptional level and by VHL $\alpha$ through feedback at post-translational level. Here we sought to address the oncogenic properties of hnRNPA2B1 in the context of cmyc and VHL $\alpha .786-0$ cells were chosen for the following investigation in view of its intrinsic mutation of VHL and loss-of-function as E3 ligase to exclude potential interference of hnRNPA2B1-VHL $\alpha$ feedback loop. We first established stable cell with ectopic c-myc alone or in combination with either shhnRNPA2B1 or VHL $\alpha$, and ectopic hnRNPA2B1 alone or in combination with VHL $\alpha$. The success in establishments of stable cells were validated at both transcript level by quantitative PCR (Fig. 5a) and protein level by western blots (Fig. 5b). We noticed despite of high level of hnRNPA2B1 mRNA in c-myc and VHL $\alpha$ double-proficient cells, the protein level of hnRNPA2B1 was relatively low which was consistent with VHL $\alpha$-mediated degradation mechanism. Ectopic introduction of c-myc significantly stimulated cell viability in comparison with empty control, which was greatly compromised by shRNA-mediated knockdown of hnRNPA2B1 (Fig. 5c) and further suppressed by cotransfection with VHL $\alpha$. Likewise, introduction of hnRNPA2B1 alone was sufficient to upregulate cell viability, which was completely blockaded by VHL $\alpha$. These results indicated the important roles of hnRNPA2B1 in mediating the pro-tumoral activities of c-myc, which was under stringent control by VHL $\alpha$ feedback loop. Notably, the greater inhibitory effects of VHL $\alpha$ than hnRNPA2B1specific shRNA suggested the other anti-tumor mechanisms of VHL $\alpha$ such as inhibition of hypoxia induced factor 


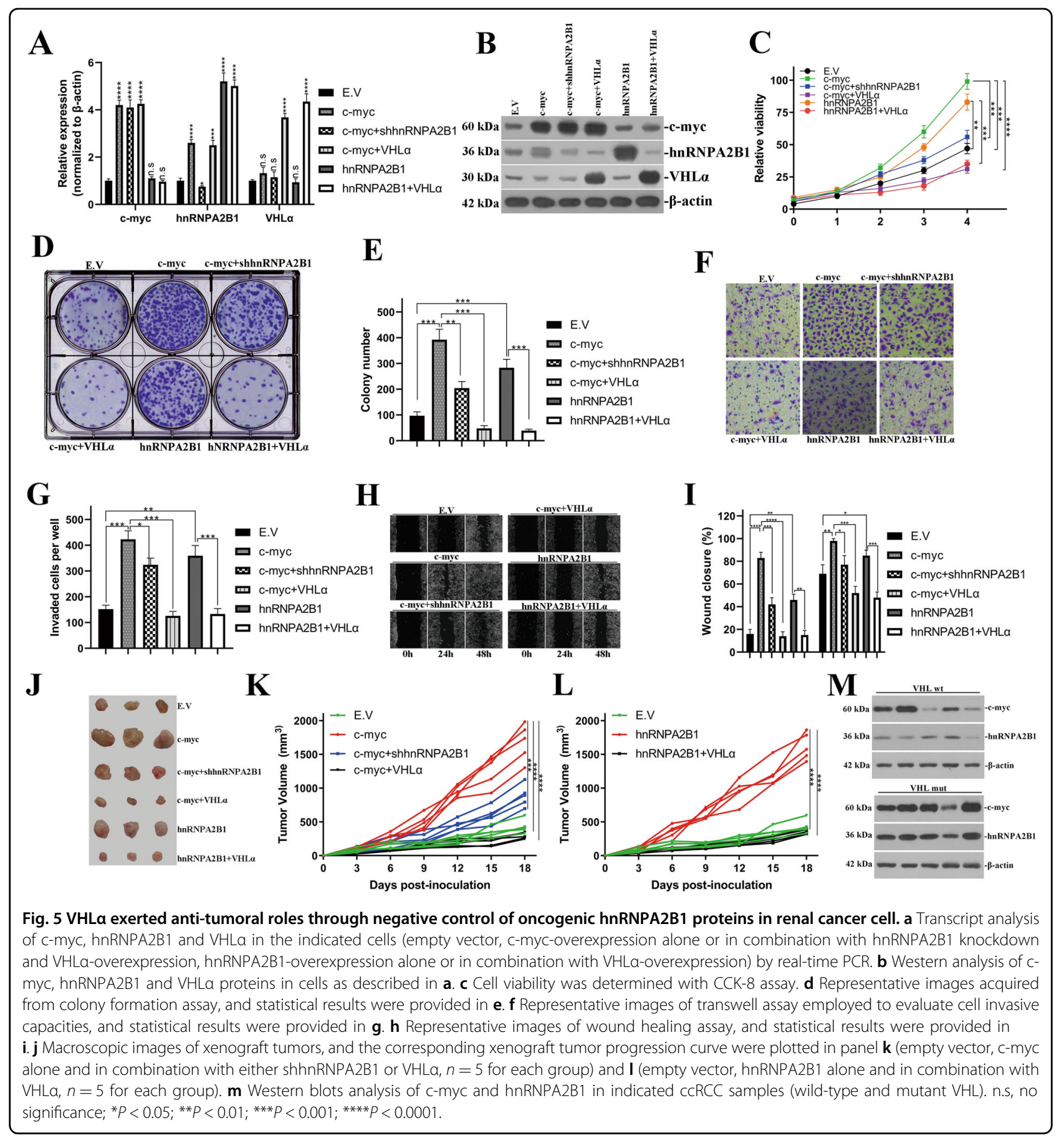

as previously described ${ }^{18}$. The pro-proliferative activities of hnRNPA2B1 was also uncovered by colony formation assay, wherein c-myc overexpression dramatically increased the colony number which was greatly reduced by either hnRNPA2B1 depletion or VHL $\alpha$-proficiency (representative images and statistical data were provided in Fig. 5d, e, respectively). Complementation with VHL $\alpha$ also completely inhibited colony formation stimulated by hnRNPA2B1 overexpression. The metastatic capacities were measured as well with both transwell assay and wound healing assay. As shown in Fig. 5f, g, c-myc significantly induced transwell capacities in comparison with empty vector control, which was partially inhibited by hnRNPA2B1 silencing while completely abolished by cotransfection with VHL $\alpha$. Introduction of hnRNPA2B1 alone also promoted invasive behaviors while blockaded by VHL $\alpha$. Consistently, the migrative measurement with wound healing demonstrated that ectopic c-myc 
significantly accelerated gap closure, which was slowed by hnRNPA2B1 silencing and further decelerated by VHL $\alpha$. Transfection with hnRNPA2B1 alone imposed stimulatory effects on wound healing, and completely blockaded by ectopic VHL $\alpha$ (Fig. 5h, i). Most importantly, the oncogenic properties of hnRNPA2B1 downstream c-myc signaling and anti-tumoral feedback loop between hnRNPA2B1 and VHL $\alpha$ were consolidated with xenograft tumor in vivo. Forced overexpression of c-myc greatly promoted tumor progression, which was partially compromised by hnRNPA2B1 deficiency and completely abrogated by VHL $\alpha$. HnRNPA2B1 proficiency alone also significantly contributed to xenograft tumor growth, and similarly inhibited by complementation of VHLa (macroscopic images of xenograft tumors were shown in Fig. $5 j$, and growth curve of xenograft tumor in the context of c-myc overexpression in combination with hnRNPA2B1 knockdown and ectopic VHL $\alpha$ was provided in Fig. 5k, growth curve of xenograft tumor in the context of hnRNPA2B1 overexpression in combination with VHL $\alpha$ overexpression was shown in Fig. 5l). We further analyzed functional relevance of VHL $\alpha$, hnRNPA2B1 and c-myc in ccRCC tissue samples, and noticed relatively high abundance of hnRNPA2B1 in Myc+/VHLmt patients in comparison with Myc+/VHLwt ones (Fig. $5 \mathrm{~m}$ ). Our data demonstrated the oncogenic properties of hnRNPA2B1 especially in the context of c-myc signaling, and antagonized by its regulated VHL $\alpha$.

\section{Discussion}

Our previous study identified a novel alternative translation isoform of VHL $\alpha$ and preliminarily characterized its anti-tumor properties in renal cancer. Here we further unraveled its CUG-mediated initiation and subjection to both eukaryotic initiator factor eIF2A and RNA helicase eIF4A regulations. As expected, employments of ATGinitiating inhibitor ATA significantly induced VHL $\alpha$ translation, while CUT-initiating inhibitor acriflavine suppressed VHL $\alpha$ ratio at protein level. Via systematic knockdown method, we uncovered that eIF4A as the main RNA helicase involving in VHL $\alpha$ translation. Notably, we examined available eIF4A inhibitors including pateamine A, hippuristanol and silvestrol, all of which manifested significant inductions on VHL $\alpha$ translation and worthied further investigation into the potential antitumoral activities in this regard.

Our previous study also uncovered that VHL $\alpha$ negatively regulated hnRNPA2B1 and eventually modulated pyruvate kinase transcript splicing and reprogramed cellular glucose metabolism in renal cancer cells. Here we further identified the ubiquitination of hnRNPA2B1 at lysine residue 174 and 305 by VHL $\alpha$, which critically depended on the amino acid 1-38 of VHL $\alpha$. We speculated that this fragment which was unique to VHL $\alpha$ was responsible for both specificity and affinity to hnRNPA2B1, and therefore endowed VHL $\alpha$ other than VHL-24 and VHL-19 to mediate hnRNPA2B1 ubiquitination and degradation through proteasome pathway. Via retrieval of the Catalogue of Somatic Mutations in Cancer database (COSMIC, cancer.sanger.ac.uk/cosmic), we noticed that $-134 \mathrm{G}>\mathrm{C}$ point mutation locating in the $\mathrm{N}$ terminal of VHL $\alpha$ protein caused arginine to proline transition and therefore might disrupt interaction between VHL $\alpha$ and hnRNPA2B1 and VHL $\alpha$-mediated hnRNPA2B1 degradation through ubiquitin-proteasome pathway. According to the subcellular localization as indicated by confocal images, VHL $\alpha$-mediated ubiquitination of hnRNPA2B1 predominantly occurred in cell nucleus where hnRNPA2B1 exerted its primary biological function as heterogenous nuclear ribonucleoprotein.

Notably, in this study we unexpectedly found that VHL $\alpha$ other than VHL-19 and VHL-24 was transiently induced by ectopic hnRNPA2B1, which implicated the involvement of post-transcriptional mechanism in this scenario. In view of its intrinsic properties as RNAbinding protein, here we provided experimental evidence in support of the direct interaction between hnRNPA2B1 protein with VHL transcripts. We noticed both VHL-24 and VHL $\alpha$ were capable of complexing with hnRNPA2B1, which was absent in the VHL-19-pulldown protein species. The unusual octadic pentamer sequence predominantly mediated this interaction, and our results demonstrated that VHL $\alpha$ translation enhanced by ectopic hnRNPA2B1 was partially blockaded by mutation introduced into pentamer- 5 and pentamer- 8 both individually or in combination, and further completely abrogated by scramble mutation of whole octadic pentamer sequences. In this regard, we speculated that mutations curated in COSMIC database which located in pentamer region might manifest similar phenotype and abrogate the inducing effect of hnRNPA2B1 on VHL alternative translation. The delicate regulation and potential involvement of eIF4A in this scenario was still to be experimentally clarified.

The negative regulation of VHL $\alpha$ on hnRNPA2B1 via ubiquitination-proteasome degradation and positive regulation of hnRNPA2B1 on VHL $\alpha$ alternative translation herein constituted a feedback loop, which appeared to consequently contribute to self-limiting of hnRNPA2B1 in the context of upstream oncogenic signaling such as cmyc. In agreement with previous report, here we described c-myc-mediated regulation of hnRNPA2B1 transcription in renal cancer cells ${ }^{26}$. We would like to emphasize the distinct finding of our study in respect of cmyc-recognizing site in hnRNPA2B1 promoter in renal cancer. Via bioinformatic prediction and experimental validation, we identified $-281 \sim 275$ nt fragment as ciselement of c-myc and mutation of which greatly abolished 
the luciferase reporter activity of hnRNPA2B1. This regulation was further consolidated by the observation of significant positive correlation between c-myc and hnRNPA2B1 in kidney renal clear cell carcinomas curated in the TCGA database and in our renal tumor collection. Our data suggested upregulated hnRNPA2B1 as immediate events downstream aberrant c-myc signaling, which was subsequently subjected to the control of VHL $\alpha$. Therefore, hnRNPA2B1 highly likely exert oncogenic functions in the context of c-myc activation and VHL $\alpha$ disruption in renal cancer. The current results suggested the specificity of VHL $\alpha$ exerting anti-tumoral function against activated hnRNPA2B1 in renal tumors, and therefore somatic mutations in VHL $\alpha$ which interrupted the feedback loop might compromise its tumor suppressor activity, which worthied further investigations to fully understand the novel isoform of VHL.

Our study also uncovered the pro-tumoral activities of hnRNPA2B1 in renal cancer cells. We found that introduction of c-myc tremendously stimulated cell proliferation, migration, invasion and xenograft tumor progression. This was in agreement with some previous reports. Tang et al. disclosed that myc signaling network was significantly activated and fundamental to the cell proliferation of clear cell renal cell carcinoma ${ }^{27}$. A conditional transgenic mouse model established by Shroff et al. demonstrated that myc oncogene overexpression was sufficient to initiate and maintain renal cell carcinoma, which critically upregulated glutaminolytic pathway instead of glycolytic pathway ${ }^{28}$. Our data suggested the oncogenic roles of c-myc was partially mediated by hnRNPA2B1 and greatly antagonized by VHL $\alpha$, which was consistent with previous study showing that myc activation cooperated with VHL and INK4A/ARF loss in induction of clear cell renal cell carcinoma ${ }^{29}$. The insufficiency of myc in tumorigenesis in kidney with wild-type VHL implicated in this study was consolidated by our observations both in vitro and in vivo. We proposed that myc activation accelerated accumulation of hnRNPA2B1 in the context of VHL loss, which convergently contributed to the tumorigenesis. The tumorigenic activities of hnRNPA2B1 in VHL-deficient cells were also disclosed with stimulatory effects on cell proliferation, migration, invasion and xenograft tumor growth in our results, which was previously proposed by several investigations. For instance, Dowling et al. showed the abnormal levels of hnRNPA2B1 in solid tumors and peripheral blood from diagnosed lung cancer patients ${ }^{30}$. Through interaction with and regulation of oncogenic KRAS, hnRNPA2B1 was suggested involving in tumorigenesis of pancreatic ductal adenocarcinoma ${ }^{31}$. Therefore, our results unraveled a feedback loop between hnRNPA2B1 and VHL $\alpha$, which exerted tumor suppressor roles downstream aberrant cmyc signaling.
In summary, our results demonstrated critical involvements of eIF2A and eIF4A in VHL $\alpha$ alternative translation, which specifically target hnRNPA2B1 to constitute a feedback loop and imposed stringent control on hnRNPA2B1 protein even in the context of c-myc aberrantly activated. In addition, we proposed therapeutic potentials of eIF4A inhibitors against renal cancer with intact VHL gene, which definitely worthied further investigations.

\section{Acknowledgements \\ This work was supported by National Natural Science Foundation of China, No. 81672796 to Y.B.L. \\ Author details \\ 'Institute of Immunology and Molecular Medicine, Jining Medical University, Jining, China. 'Department of Urological Surgery, Longgang District People's Hospital of Shenzhen, Shenzhen, China. ${ }^{3}$ Department of Biochemistry, \\ Zhongshan School of Medicine, Sun Yat-sen University, Guangzhou, China}

\section{Data availability}

All data generated or analyzed during this study are included in this published article.

\section{Ethics approval and consent to participate}

This study was approved by the Jining Medical University Institutional Review Board.

\section{Consent for publication}

All authors are aware and agree to the content of the paper and their authorships.

\section{Conflict of interest}

The authors declare that they have no conflict of interest.

\section{Publisher's note}

Springer Nature remains neutral with regard to jurisdictional claims in published maps and institutional affiliations.

Received: 24 March 2020 Revised: 29 July 2020 Accepted: 29 July 2020 Published online: 11 August 2020

\footnotetext{
References

1. Gnarra, J. R. et al. Mutations of the VHL tumour suppressor gene in renal carcinoma. Nat. Genet. 7, 85-90 (1994).

2. Latif, F. et al. Identification of the von Hippel-Lindau disease tumor suppressor gene. Science 260, 1317-1320 (1993).

3. Zbar, B., Brauch, H., Talmadge, C. \& Linehan, M. Loss of alleles of loci on the short arm of chromosome 3 in renal cell carcinoma. Nature 327, 721-724 (1987).

4. Young, A. C. et al. Analysis of VHL gene alterations and their relationship to clinical parameters in sporadic conventional renal cell carcinoma. Clin. Cancer Res. 15, 7582-7592 (2009).

5. Cancer Genome Atlas Research, N. Comprehensive molecular characterization of clear cell renal cell carcinoma. Nature 499, 43-49 (2013).

6. Kibel, A., Iliopoulos, O., DeCaprio, J. A. \& Kaelin, W. G. Jr Binding of the von Hippel-Lindau tumor suppressor protein to Elongin B and C. Science 269, 1444-1446 (1995).

7. Kamura, T. et al. Rbx1, a component of the VHL tumor suppressor complex and SCF ubiquitin ligase. Science 284, 657-661 (1999).

8. Pause, A. et al. The von Hippel-Lindau tumor-suppressor gene product forms a stable complex with human CUL-2, a member of the Cdc53 family of proteins. Proc. Natl Acad. Sci. USA 94, 2156-2161 (1997).
} 
9. Maxwell, P. H. et al. The tumour suppressor protein $\mathrm{VHL}$ targets hypoxiainducible factors for oxygen-dependent proteolysis. Nature 399, 271-275 (1999).

10. Semenza, G. L. Hypoxia-inducible factors: mediators of cancer progression and targets for cancer therapy. Trends Pharm. Sci. 33, 207-214 (2012).

11. Gnarra, J. R. et al. Post-transcriptional regulation of vascular endothelial growth factor mRNA by the product of the VHL tumor suppressor gene. Proc. Natl Acad. Sci. USA 93, 10589-10594 (1996).

12. Sendoel, A., Kohler, I., Fellmann, C., Lowe, S. W. \& Hengartner, M. O. HIF-1 antagonizes p53-mediated apoptosis through a secreted neuronal tyrosinase Nature 465, 577-583 (2010).

13. Escudier, B., Szczylik, C., Porta, C. \& Gore, M. Treatment selection in metastatic renal cell carcinoma: expert consensus. Nat. Rev. Clin. Oncol. 9 327-337 (2012).

14. Blankenship, C., Naglich, J. G., Whaley, J. M., Seizinger, B. \& Kley, N. Alternate choice of initiation codon produces a biologically active product of the von Hippel Lindau gene with tumor suppressor activity. Oncogene 18, 1529-1535 (1999).

15. Hiopoulos, O., Ohh, M. \& Kaelin, W. G. Jr pVHL19 is a biologically active product of the von Hippel-Lindau gene arising from internal translation initiation. Proc. Natl Acad. Sci. USA 95, 11661-11666 (1998).

16. Schoenfeld, A., Davidowitz, E. J. \& Burk, R. D. A second major native von Hippel-Lindau gene product, initiated from an internal translation start site, functions as a tumor suppressor. Proc. Natl Acad. Sci. USA 95, 8817-8822 (1998).

17. Liu, Y., Yang, H., Zuo, F. \& Chen, L. The VHL short variant involves in protein quality control. Gene 589, 63-71 (2016).

18. Liu, Y. et al. A novel VHLalpha isoform inhibits Warburg effect via modulation of PKM splicing. Tumour Biol. 37, 13649-13657 (2016).

19. Starck, S. R. et al. Leucine-tRNA initiates at CUG start codons for protein synthesis and presentation by MHC class I. Science 336, 1719-1723 (2012).
20. Liang, $H$. et al. PTENalpha, a PTEN isoform translated through alternative initiation, regulates mitochondrial function and energy metabolism. Cell Metab. 19, 836-848 (2014).

21. Dasso, M. C. \& Jackson, R. J. Efficient initiation of mammalian mRNA translation at a CUG codon. Nucleic Acids Res. 17, 6485-6497 (1989).

22. Goodarzi, $H$. et al. Systematic discovery of structural elements governing stability of mammalian messenger RNAs. Nature 485, 264-268 (2012).

23. Low, W. K. et al. Inhibition of eukaryotic translation initiation by the marine natural product pateamine A. Mol. Cell 20, 709-722 (2005).

24. Tsumuraya, T. et al. Effects of hippuristanol, an inhibitor of elF4A, on adult Tcell leukemia. Biochem. Pharm. 81, 713-722 (2011).

25. Nguyen, T. M. et al. FGFR1-activated translation of WNT pathway components with structured $5^{\prime}$ UTRs is vulnerable to inhibition of ElF4A-dependent translation initiation. Cancer Res. 78, 4229-4240 (2018).

26. David, C. J., Chen, M., Assanah, M., Canoll, P. \& Manley, J. L. HnRNP proteins controlled by c-Myc deregulate pyruvate kinase mRNA splicing in cancer. Nature 463, 364-368 (2010).

27. Tang, S. W. et al. MYC pathway is activated in clear cell renal cell carcinoma and essential for proliferation of clear cell renal cell carcinoma cells. Cancer Lett. 273, 35-43 (2009).

28. Shroff, E. H. et al. MYC oncogene overexpression drives renal cell carcinoma in a mouse model through glutamine metabolism. Proc. Natl Acad. Sci. USA 112 6539-6544 (2015).

29. Bailey, S. T. et al. MYC activation cooperates with Vhl and Ink4a/Arf loss to induce clear cell renal cell carcinoma. Nat. Commun. 8, 15770 (2017).

30. Dowling, P. et al. Abnormal levels of heterogeneous nuclear ribonucleoprotein A2B1 (hnRNPA2B1) in tumour tissue and blood samples from patients diagnosed with lung cancer. Mol. Biosyst. 11, 743-752 (2015).

31. Barcelo, C. et al. Ribonucleoprotein HNRNPA2B1 interacts with and regulates oncogenic KRAS in pancreatic ductal adenocarcinoma cells. Gastroenterology 147, 882-892 e888 (2014). 